\title{
Hydroxychloroquine protects melanocytes from autoantibody-induced injury by reducing the binding of antigen-antibody complexes
}

\author{
DONG-GUANG LI ${ }^{1 *}$, WEN-ZHI HU ${ }^{1 *}$, HUI-JUN MA ${ }^{2}$, WEN LIU ${ }^{1}$, QING-QI YANG ${ }^{1}$ and GUANG ZHAO ${ }^{1}$ \\ ${ }^{1}$ Department of Dermatology, The General Hospital of Air Force, Beijing 100142; ${ }^{2}$ Department of Dermatology, \\ The 309th Hospital of Chinese PLA, Beijing 100091, P.R. China
}

Received June 16, 2015; Accepted May 25, 2016

DOI: $10.3892 / \mathrm{mmr} .2016 .5354$

\begin{abstract}
Vitiligo is a polygenic autoimmune disorder characterized by loss of pigmentation due to melanocyte destruction. Hydroxychloroquine (HCQ) is an effective immunosuppressant widely used in the treatment of autoimmune disorders. As generalized vitiligo $(\mathrm{GV})$ is commonly considered to be a $\mathrm{T}$ cell and autoantibody-induced immune disorder, the present study aimed to determine whether HCQ protects melanocytes from autoantibody-induced disruption. Anti-melanocyte antibodies were obtained from the serum of patients with progressive $\mathrm{GV}$ and the effects of HCQ on prevent the autoantibody-induced disruption of melanocytes was observed. Cell-based ELISA, indirect immunofluorescence and western blotting were used to analyze the autoantibody content of sera samples obtained from 32 patients with progressive GV. The cytotoxicity of HCQ was detected by MTT assay, and $1 \mu \mathrm{g} / \mathrm{ml} \mathrm{HCQ}$ was applied to human primary melanocytes (HMCs) to examine whether it could exert protective effects against autoantibody-induced immune injury. Flow cytometry was used to measure autoantibody binding to the surface of HMCs. Complement-dependent cytotoxicity (CDC) and antibody-dependent cell-mediated cytotoxicity (ADCC) were monitored by MTT and lactate dehydrogenase-releasing assays. The concentration of autoantibodies in sera samples taken from GV patients was significantly higher than in controls, particularly in patients who had $>10 \%$ of their body
\end{abstract}

Correspondence to: Dr Hui-Jun Ma, Department of Dermatology, The 309th Hospital of Chinese PLA, A17 Heishanhu Road, Haidian, Beijing 100091, P.R. China

E-mail: melanocytes@163.com

Dr Guang Zhao, Department of Dermatology, The General Hospital of Air Force, 30 Fucheng Road, Haidian, Beijing 100142, P.R. China E-mail: guangfen@yahoo.com

${ }^{*}$ Contributed equally

Key words: melanocytes, hydroxychloroquine, autoantibody, antigen-antibody complexes surface affected by vitiligo. The majority of the autoantibodies presented in the HMCs and human keratinocytes (HKCs) and were predominantly localized to the cell surface and cytoplasm. The molecular weights of the autoantigens were identified as 30, 37-39, 42, 53, 60-75, 90, 100, 110, and $126 \mathrm{kDa}$; the $30 \mathrm{kDa}$ protein was observed only in HMCs. The addition of HCQ at a concentration of $1 \mu \mathrm{g} / \mathrm{ml}$ produced no significant cytotoxicity in HMCs and was demonstrated to reduce the binding of $\mathrm{GV}$ immunoglobulin $\mathrm{G}$ ( $\mathrm{IgG}$ ) to the surface of HMCs. HCQ also significantly decreased the effects of ADCC and CDC that were mediated by GV IgG. The present study provides evidence that HCQ dissociates autoantibody-antigen complexes on the surface of HMCs and reverses ADCC and CDC activity in vitro. Thus, in addition to its effectiveness as an antimalarial therapeutic agent, HCQ may also be a promising potential treatment for patients with vitiligo.

\section{Introduction}

Vitiligo is an acquired multifactorial depigmenting disorder characterized by the appearance of white macules in the skin in response to the progressive loss of functional melanocytes within the epidermis (1). A number of types of vitiligo are distinguished according to the distribution patterns of the lesions. Generalized vitiligo $(\mathrm{GV})$ is the predominant type and is characterized by multiple scattered lesions that form a symmetrical distribution pattern. GV is often progressive and patients exhibit phases of stabilized depigmentation, an extending vitiligo with enlarging lesions, or the development of new lesions, which is defined as active vitiligo (2). Three major hypotheses have been proposed to explain the pathogenesis of vitiligo: Autoimmune injury, oxidative stress and the neural hypothesis. GV is commonly hypothesized to arise in response to autoimmune injury and is the type of vitiligo that was focused on in the present study. Two additional dysfunctions of the immune system have been proposed to result in the development of autoimmune-associated vitiligo, a $\mathrm{T}$ cell-based pathomechanism in cases of localized disease and T cells, NK cells and antibodies have been implicated in the pathogenesis of diffuse vitiligo (3).

Hydroxychloroquine (HCQ) is an antimalarial compound that has been demonstrated as an effective immunosuppressive 
therapeutic agent, it is widely applied in the treatment of systemic lupus erythematosus (SLE) and rheumatoid arthritis (RA) (4). Considering the high protein-binding capacity of HCQ (4), the present study aimed to determine whether HCQ may directly affect the formation of antigen-antibody complexes and whether disturbed autoantibodies mediate either complement-dependent cytotoxicity (CDC) and/or antibody-dependent cell-mediated cytotoxicity (ADCC) activity. The present study measured the effects of self-reactive antibodies in progressive GV patients and investigated the protection offered by HCQ in reducing antigen-antibody complex binding.

\section{Materials and methods}

Ethics statement. The present study was approved by the ethics committee of The General Hospital of Air Force (Beijing, China). All participants provided written informed consent prior to participation in the study.

Patients. The present study included 32 progressive GV patients who were recruited based on the following criteria: i) A clinical diagnosis of GV in accordance with associated criteria (5); ii) no use of any systemic therapy for $\geq 4$ weeks prior to enrollment in the study; and iii) the development of new lesions within the 3 months preceding study enrollment. Unaffected individuals $(n=27)$ were recruited as controls. The clinical characteristics of the patients and controls are summarized in Table I.

Peripheral blood samples. For serological studies, $10 \mathrm{ml}$ of peripheral blood was drawn from each subject and stored at $-80^{\circ} \mathrm{C}$. Human peripheral blood mononuclear cells (PBMCs) were isolated from the heparinized blood of healthy volunteer donors via centrifugation over a Ficoll-Hypaque density gradient (TBD Science, Tianjin, China).

Cell culture and propagation. Human primary melanocytes (HMCs) and human keratinocytes (HKCs) were obtained from the unaffected skin of each patient. Fresh skin biopsies were rinsed 3 times in phosphate-buffered saline (PBS; pH 7.4) containing antibiotics $(5,000 \mathrm{U} / \mathrm{ml}$ penicillin and $5 \mathrm{mg} / \mathrm{ml}$ streptomycin), cut into small pieces $(5 \times 5 \mathrm{~mm})$, and incubated for $14 \mathrm{~h}$ at $4^{\circ} \mathrm{C}$ in Dulbecco's modified Eagle's medium (Gibco; Thermo Fisher Scientific, Inc., Waltham, MA, USA) containing dispase ( $2 \mathrm{mg} / \mathrm{ml}$; Roche Diagnostics, Basel, Switzerland). The epidermis was separated from the dermis using sterile forceps. The cells of the epidermis were then isolated using $0.05 \%$ trypsin with 2 mM EDTA (Nanjing KeyGen Biotech Co., Ltd., Nanjing, China). The protocols used to culture HMCs and HKCs were slightly modified from our previously described method (6). Briefly, cells were adjusted to $2.5 \times 10^{4}$ cells $/ \mathrm{cm}^{2}$ following washing in PBS and then cultured in either M-254 medium (Gibco; Thermo Fisher Scientific, Inc.) for HMCs or EpiLife medium (Gibco; Thermo Fisher Scientific, Inc.) for HKCs. The cell cultures were maintained at $37^{\circ} \mathrm{C}$ in a humidified $5 \% \mathrm{CO}_{2}$ atmosphere and the medium was changed at 3-4 day intervals. When cells reached 70-90\% confluence they were split using trypsin-EDTA (Nanjing KeyGen Biotech Co., Ltd.) treatment for $5 \mathrm{~min}$ at room temperature and inactivated
Table I. Clinical characteristic of vitiligo patients and healthy controls.

\begin{tabular}{lcc}
\hline Characteristic & Healthy controls & GV patients \\
\hline Sex (male/female) & $12 / 15$ & $16 / 16$ \\
Age, median (range) & $30(24-63)$ & $25(9-61)$ \\
$\begin{array}{l}\text { Months since } \\
\text { diagnosis median (range) }\end{array}$ & $\mathrm{ND}$ & $54(4-360)$ \\
$\begin{array}{l}\text { Intensity \%, median (range) } \\
\text { GV, generalized vertiligo; ND, no data. }\end{array}$ & $15(0.1-80)$ \\
\hline
\end{tabular}

with fetal bovine serum (TBD Science). HMC and HKC populations were considered pure following 3-4 passages.

Cell-based ELISA. HMCs were detached and seeded on 96-well cell culture plates at a density of 40,000 cells/well. Following incubation for $24 \mathrm{~h}$, cells were fixed in $4 \%$ paraformaldehyde (PFA) for $15 \mathrm{~min}$ at room temperature. Fixed cells were then washed 3 times with PBS and blocked with $100 \mu 15 \%$ bovine serum albumin (BSA; Solarbio Science \& Technology Co., Ltd., Beijing, China) in PBS for $1 \mathrm{~h}$ at $37^{\circ} \mathrm{C}$. Following this, supernatants were removed and the cells were incubated with $100 \mu \mathrm{l}$ diluted serum obtained from healthy volunteers or from vitiligo patients (diluted 1:10 in 5\% BSA in PBS) at $37^{\circ} \mathrm{C}$ for $1 \mathrm{~h}$. The plates were washed 3 times with PBS and incubated with horseradish peroxidase (HRP)-conjugated goat anti-human immunoglobulin $\mathrm{G}(\mathrm{IgG})$ heavy and light chains (H+L) antibody (cat. no. ZB-2304; ZSGB-BIO, Beijing, China; dilution 1:10,000 in PBS with 5\% BSA) for $1 \mathrm{~h}$ at $37^{\circ} \mathrm{C}$. Following washing three times in PBS, the results were visualized using tetramethylbenzidin substrate solution (Beyotime Institute of Biotechnology, Haimen, China). The enzymatic reaction was then arrested with sulfuric acid and the intensity of the resulting color was determined by measuring absorbance at a wavelength of $450 \mathrm{~nm}\left(\mathrm{~A}_{450}\right)$ using microplate reader (RT-600; Rayto, Shenzhen, China). The cutoff value for a positive reaction was based on the $\mathrm{A}_{450}$ values obtained from 12 healthy controls and calculated as the mean $+2.0 \mathrm{x}$ standard deviation.

Indirect immunofluorescence assay. HMCs and HMKs were seeded onto glass coverslips in 6-well culture plates and fixed with $4 \%$ PFA for $10 \mathrm{~min}$ at room temperature. The cells were then washed with PBS and blocked with 5\% BSA in PBS for $30 \mathrm{~min}$ at $37^{\circ} \mathrm{C}$. Following blocking, the cells were stained with either patient or control serum (diluted 1:10 in 5\% BSA in PBS) or the mouse anti-CK (pan) monoclonal antibody (cat. no. ZN-0069; diluted 1:100 in 5\% BSA in PBS; ZSGB-BIO) for $30 \mathrm{~min}$ at $37^{\circ} \mathrm{C}$. Cells were then washed and incubated with either fluorescein isothiocyanate (FITC)-conjugated goat anti-human IgG (H+L) antibody (cat. no. ZF-0308; 1:200; ZSGB-BIO) or tetramethylrhodamine-conjugated goat anti-mouse IgG $(\mathrm{H}+\mathrm{L})$ antibody (cat. no. ZF-0313; 1:200; ZSGB-BIO) for $30 \mathrm{~min}$ at $37^{\circ} \mathrm{C}$. Following three washes in PBS, the cells were observed by fluorescence microscopy.

Detecting autoantibodies using western blotting. HMCs and HKCs were grown in 100-mm culture dishes and protein 
extraction was extracted using RIPA lysis buffer on ice for $10 \min [150 \mathrm{mM} \mathrm{NaCl}, 1 \%$ Triton X-100, $50 \mathrm{mM}$ Tris- $\mathrm{HCl}$ ( $\mathrm{pH} 7.4$ ), $0.5 \%$ sodium dezoxycholate, $0.1 \%$ SDS and sodium orthovanadate, sodium fluoride, EDTA (pH 7.4); Applygen Technologies, Inc., Beijing, China] containing protease inhibitors (Applygen Technology, Inc.). The total protein concentration of the lysate was estimated using a BCA protein assay kit (Beijing Solarbio Science \& Technology Co., Ltd., Beijing, China). The extracts were mixed with loading buffer (Applygen Technologies, Inc.) and incubated at $100^{\circ} \mathrm{C}$ for $10 \mathrm{~min}$, following which they were electrophoresed on a $10 \%$ SDS-polyacrylamide gel. A $20 \mu \mathrm{g}$ aliquot of each sample was loaded in each lane. The separated proteins were electrotransferred onto a polyvinylidene difluoride membrane (EMD Millipore, Billerica, MA, USA), which was then blocked using 5\% BSA in Tris-buffered saline and Tween 20 (TBST; pH 7.4). The membrane was probed with either control or patient serum (diluted 1:25 in 5\% BSA in TBST) or a mouse anti- $\beta$-actin monoclonal antibody (cat. no. TA-09; diluted 1:1,000 in 5\% BSA in TBST; ZSGB-BIO), overnight at $4^{\circ} \mathrm{C}$. The blot was then incubated with either an HRP-conjugated goat anti-human IgG monoclonal antibody (ZSGB-BIO) or an HRP-conjugated goat anti-mouse IgG monoclonal antibody (ZSGB-BIO) at a dilution of 1:20,000 (in 5\% BSA in TBST) for $1 \mathrm{~h}$ at room temperature. The signals were detected using EnlightTM Western Blot Detection Reagents (Engreen Biosystem New Zealand Ltd., Auckland, New Zealand) followed by exposure to X-ray film (Kodak, Rochester, NY USA). The results were analyzed using Quantity One 4.62 software.

Separation and purification of IgG. Positive serum samples were identified based on the results obtained from the cell-based ELISA. Serum IgG fractions (isolated from 12 positive patients and normal controls) were enriched by repeated ammonium sulfate precipitation and further purified by diethylaminoethyl cellulose-52 exchange chromatography (Whatman; GE Healthcare Life Sciences, Little Chalfont, $\mathrm{UK})$. Following centrifugal ultrafiltration at 4,000 x g, for $15 \mathrm{~min}$ at $4^{\circ} \mathrm{C}, \mathrm{IgG}$ fractions were concentrated $\sim 40 \mathrm{mg} / \mathrm{ml}$ and stored at $-80^{\circ} \mathrm{C}$.

Cytotoxicity of HCQ. HCQ-induced cytotoxicity was detected using the MTT assay (Sigma-Aldrich, St. Louis, MO, USA). HMCs were seeded into 96 -well culture plates at a density of 2,000 cells/well and then incubated at $37^{\circ} \mathrm{C}$ in a humidified atmosphere with $5 \% \mathrm{CO}_{2}$ for $24 \mathrm{~h}$. The HMCs were treated with HCQ at concentrations of $0,0.5,1,2.5$, and $5 \mu \mathrm{g} / \mathrm{ml}$. Following incubation for $0,24,48$, and $72 \mathrm{~h}$, MTT solution ( $5 \mathrm{mg} / \mathrm{ml}$; Sigma-Aldrich) was added to each well and the cells were incubated for a further $4 \mathrm{~h}$. The culture medium was then removed and $100 \mu \mathrm{l}$ of dimethyl sulfoxide was added to each well to dissolve the formazan crystals. The absorbance was measured at wavelength of $490 \mathrm{~nm}\left(\mathrm{~A}_{490}\right)$. Cell viability was associated with $\mathrm{A}_{490}$ measurements.

Flow cytometry (FCM). The effects of HCQ on IgG binding to HMCs were determined using flow cytometry. IgG was obtained from patient serum samples as described above. HMCs were fixed in 4\% PFA and incubated with purified serum $\mathrm{IgG}$ at $800 \mu \mathrm{g} / \mathrm{ml}$ for $1 \mathrm{~h}$ at $37^{\circ} \mathrm{C}$, with or without
Table II. Melanocyte-specific autoantibodies in the sera of vitiligo patients and healthy controls.

\begin{tabular}{lccc}
\hline Groups & $\mathrm{n}$ & Positive $(\%)$ & $\begin{array}{c}\text { Absorbance at } \\
450 \mathrm{~nm}\end{array}$ \\
\hline Control & 12 & $0(0.00)$ & $0.30 \pm 0.15$ \\
Vitiligo & & & \\
Intensity $(\leq 10 \%)$ & 12 & $2(16.67)$ & $0.47 \pm 0.20^{\mathrm{a}}$ \\
Intensity $(>10 \%)$ & 12 & $10(83.33)$ & $0.68 \pm 0.23^{\mathrm{b}}$
\end{tabular}

${ }^{\mathrm{a}} \mathrm{P}<0.05$ vs. control; ${ }^{\mathrm{b}} \mathrm{P}<0.001$ vs control.

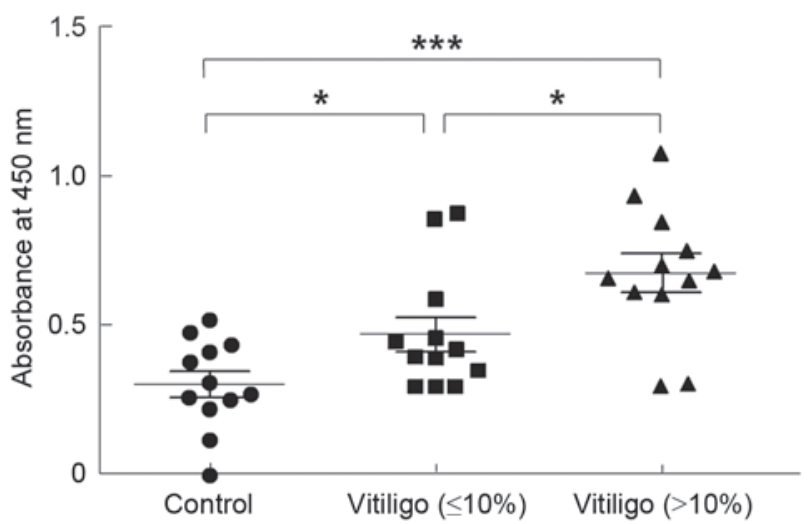

Figure 1. Distribution of absorbance values at a wavelength of $450 \mathrm{~nm}$ detected in healthy controls and in vitiligo patients. Patients were divided into two groups; one group experienced an intensity of vitiligo of $<10 \%$, and the other group experienced an intensity of $>10 \%$. ${ }^{*} \mathrm{P}<0.05,{ }^{* * * *} \mathrm{P}<0.001$.

the addition of HCQ $(1 \mu \mathrm{g} / \mathrm{ml})$. Cells were then washed and stained with FITC-labeled goat anti-human $\operatorname{IgG}(\mathrm{H}+\mathrm{L})$ antibody (1:800 dilution; ZSGB-BIO). Subsequently, the cells were washed with PBS and the samples were subjected to FCM. BD CellQuest Pro software was used to analyze the results (BD Biosciences, Franklin Lakes, NJ, USA)

CDC assay. HMCs were used to probe the CDC activity of purified immunoglobulin fractions from healthy controls or vitiligo patients. Briefly, the HMCs were seeded into 96-well culture plates at a density of 5,000 cells/well and incubated at $37^{\circ} \mathrm{C}$ for $24 \mathrm{~h}$. Subsequently, a $50 \mu \mathrm{l}$ aliquot of $\mathrm{M}-254$ medium that was premixed with various concentrations of IgG (from vitiligo patients or unaffected individuals), with or without the addition of HCQ $(1 \mu \mathrm{g} / \mathrm{ml})$, was added to each well in replicates of six and incubated at $37^{\circ} \mathrm{C}$ for $1 \mathrm{~h}$. The serum was discarded following the incubation and $50 \mu \mathrm{l}$ of normal human serum was incubated with the cells for $8 \mathrm{~h}$ at $37^{\circ} \mathrm{C}$. The MTT assay was then used to quantify the number of viable cells. Inhibition ratio $(\%)=\left(\mathrm{A}_{490}\right.$ of control group $-\mathrm{A}_{490}$ of test group) / $\mathrm{A}_{490}$ of control group x100.

ADCC assay. The ADCC activity of purified IgG was measured using a lactate dehydrogenase (LDH)-releasing assay via the CytoTox96 Non-Radioactive Cytotoxicity Assay kit (Promega Corporation, Madison, WI, USA). HMCs were incubated with 

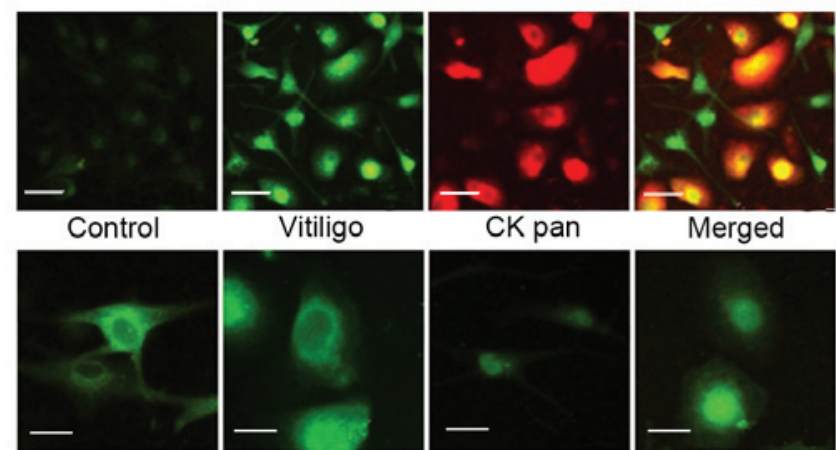

HMCs-a

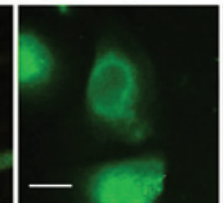

HKCs-a

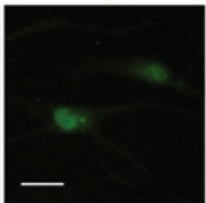

HMCs-b
Merged

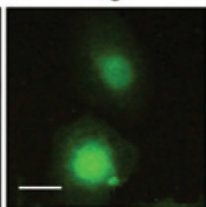

HKCs-b
Figure 2. Immunofluorescence imaging of melanocytes and keratinocytes. Melanocytes and keratinocytes were stained with serum obtained from either healthy controls or vitiligo patients. Scale bar $=80 \mu \mathrm{m}$. CK pan indicates melanocytes and keratinocytes stained with mouse anti-pan-cytokeratin antibodies; merged indicates the image was created following a merge of the vitiligo and pan-cytokeratin panels. HMCs-a and $-\mathrm{b}$ are melanocytes stained with serum obtained from vitiligo patients (scale bar $=80 \mu \mathrm{m}$ ). HKCs-a and -b are keratinocytes stained with serum obtained from vitiligo patients (scale bar $=40 \mu \mathrm{m})$.

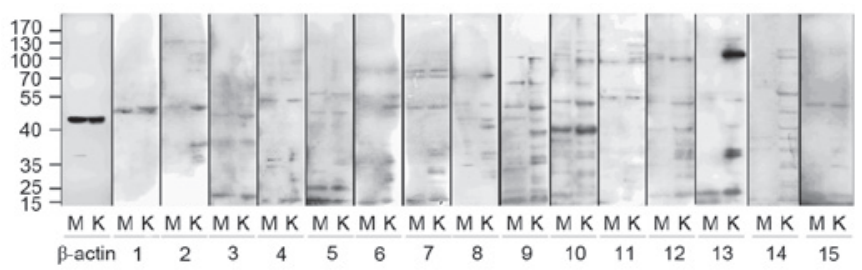

Figure 3. Western blots of serum samples obtained from patients with vitiligo demonstrating the reactivity of immunoglobulin $\mathrm{G}$ antibodies obtained from different electrophoretic fractions to melanocyte or keratinocyte extracts. Lanes $1-15$, primary antibodies were patient serum. M, extract of melanocytes; K, extract of keratinocytes.

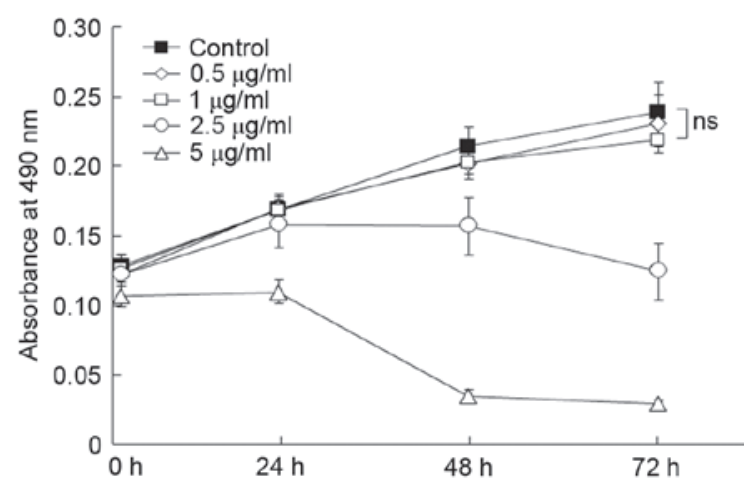

Figure 4. MTT assay results of melanocyte cytoxicity following treatment with different concentrations of hydroxychloroquine at $0,24,48$, and $72 \mathrm{~h}$. The absorbance values at a wavelength of $490 \mathrm{~nm}$ represent melanocyte growth. Data are presented as the mean \pm standard deviation $(n=6)$. $n s, P>0.1$.

normal $\operatorname{IgG}(400 \mu \mathrm{g} / \mathrm{ml})$, patient $\operatorname{IgG}(400 \mu \mathrm{g} / \mathrm{ml})$, patient $\operatorname{IgG}(400 \mu \mathrm{g} / \mathrm{ml})+\mathrm{HCQ}(1 \mu \mathrm{g} / \mathrm{ml})$, or media only for $1 \mathrm{~h}$ at $37^{\circ} \mathrm{C}$. Human PBMCs were then added to the HMCs to serve as effector cells [effector cell:target $(\mathrm{E}: \mathrm{T})$ ratio $=5: 1,10: 1$, 20:1]. Following an additional incubation at $37^{\circ} \mathrm{C}$ for $8 \mathrm{~h}$, the degree of cell lysis was determined by measuring the quantity of released $\mathrm{LDH}$. This value is positively associated with the optical density at a wavelength of $490 \mathrm{~nm}$. Controls included an LDH max control (target cells treated with $9 \%$ Triton $\mathrm{X}-100)$, an effector cell control (only effector cells) and a target cell control (only target cells). Inhibition ratio $(\%)=\left(\mathrm{A}_{490}\right.$ of test group - $\mathrm{A}_{490}$ of effector cells control - $\mathrm{A}_{490}$ of target cells control $) /\left(\mathrm{A}_{490}\right.$ of $\mathrm{LDH}$ max control - $\mathrm{A}_{490}$ of target cells control) $\mathrm{x} 100$.

Statistical analyses. Statistical tests were conducted using SPSS 16.0 software (IBM SPSS, Armonk, NY, USA). Relative anti-HMC autoantibody levels from patient and control groups were analyzed by one-way analysis of variance, and the LSD t-test was used to compare the CDC and ADCC activity of each group. Graphs were produced with GraphPad Prism 5 software (GraphPad Software, Inc., La Jolla, CA, USA). P<0.05 was considered to indicate a statistically significant difference.

\section{Results}

Serum autoantibodies against melanocytes were significantly increased in patients with GV. Patients were divided into two groups depending on the bodily area affected by vitiligo; the first group had an intensity of vitiligo of $<10 \%$, and the second group had an intensity of $>10 \%$. The two groups demonstrated significantly higher levels of IgG anti-HMCs autoantibodies compared with the controls $(\mathrm{P}<0.05$ and $\mathrm{P}<0.01$, respectively; Table II and Fig. 1).

The autoantigens in melanocytes and keratinocytes were localized by immunofluorescence. The present study investigated the subcellular distribution and appearance of autoantigens in HMCs and HKCs using a indirect immunofluorescence assay. Serum-positive patients $(n=6)$ and negative controls $(n=6)$ were observed. HMCs and HKMs that were incubated with patient serum samples produced brighter signals than healthy controls (Fig. 2). Furthermore, a signal was observed in the cytoplasm and cell surface of the HMCs and HKMs and reduced in the nucleus (Fig. 2; HMCs-a and HKCs-a) in 5/6 patients and 1/6 patients produced a higher nuclear signal (Fig. 2; HMCs-b and HKCs-b).

Antigens with molecular weight of $30 \mathrm{kDa}$ were preferentially expressed in HMCs. Western blotting indicated that HMCs and HKCs contained proteins that reacted with serum antibodies (Fig. 3). Compared with healthy controls, the antigens were predominantly observed to have a molecular weight of $30,37-39,42,53,75,90$ and $100 \mathrm{kDa}$, and occasionally of 60 , $65,68,85,110$ or $126 \mathrm{kDa}$. However, only the $30 \mathrm{kDa}$ antigens were preferentially expressed in HMCs, all other antigens were expressed in HMCs and HKCs. These results are summarized in Tables III and IV.

The cytotoxicity of HCQ in melanocytes in concentration-dependent. The cytotoxicity of HCQ was monitored in HMCs using the MTT assay. As presented in Fig. 4, concentration-dependent cytotoxicity was observed when the concentration of HCQ was $>2.5 \mu \mathrm{g} / \mathrm{ml}$. The cytotoxic effect was not observed when the concentration of HCQ was $<1 \mu \mathrm{g} / \mathrm{ml}$ when compared with the control $(\mathrm{P}>0.1)$. 
Table III. Positive reactions between vitiligo patient and control serum samples and a collection of electrophoretic fractions obtained from an extract of human melanocytes.

Electrophoretic fractions, $\mathrm{kDa}$

\begin{tabular}{lllllllllllllllll}
\cline { 2 - 3 } & $26-28$ & 30 & $33-35$ & $37-39$ & 42 & 50 & 53 & 60 & 65 & 68 & 75 & 85 & 90 & 100 & 110 & 126 \\
\hline
\end{tabular}

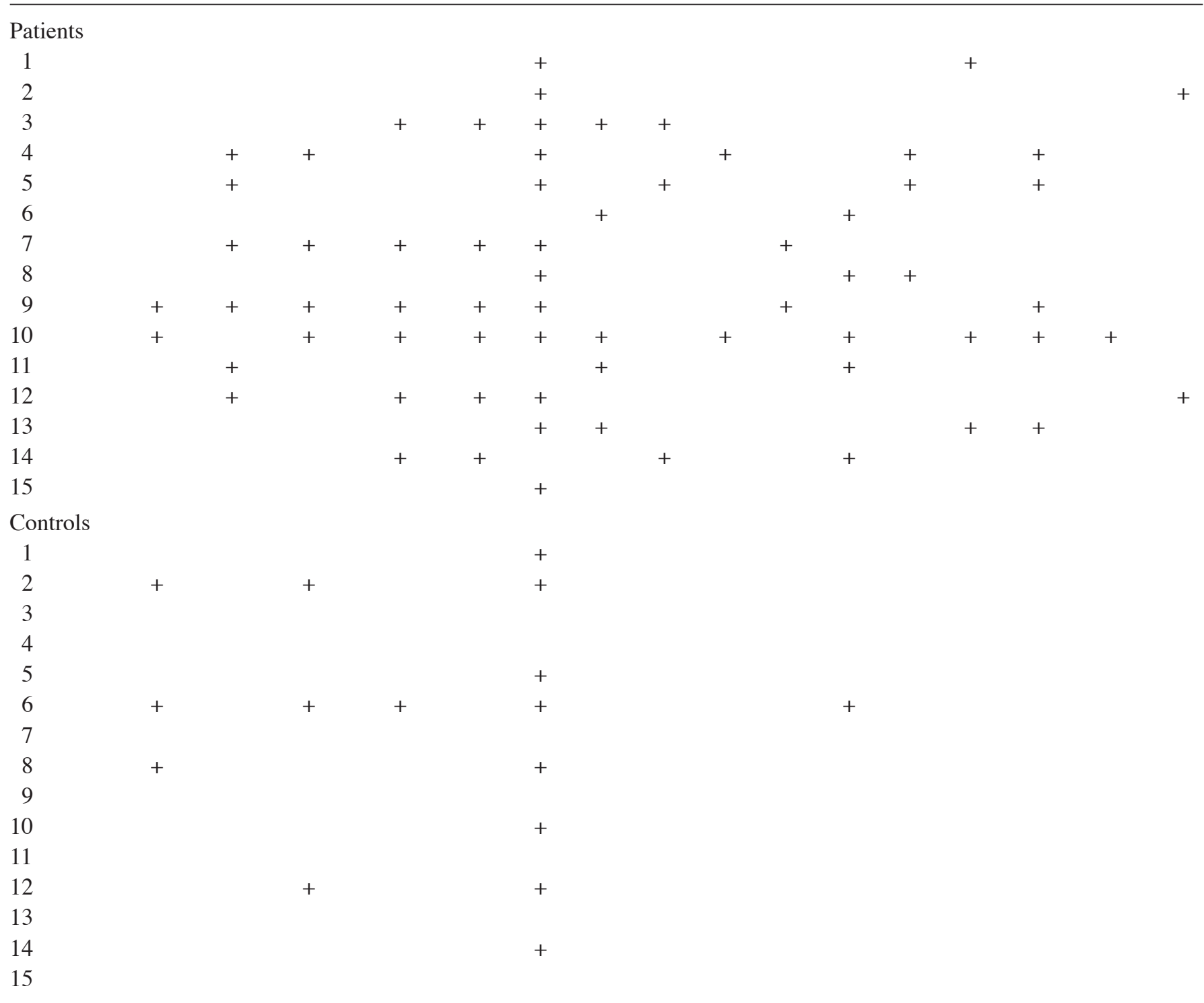

+ ,indicates a positive reaction with the corresponding fraction.

$H C Q$ reduced autoantibody binding to melanocytes. The effects of HCQ on IgG binding to HMCs were determined using the FCM assay. Incubation of HMCs with IgG samples obtained from vitiligo patients resulted in significantly greater binding of $\mathrm{IgG}$ compared with incubation with $\mathrm{IgG}$ obtained from controls $(2,962.00 \pm 35.68$ vs. $1,793.67 \pm 12.67 ; \mathrm{P}<0.001$; Fig. 5). In addition, the presence of HCQ at concentrations of $1 \mu \mathrm{g} / \mathrm{ml}$ significantly reduced the binding of $\mathrm{IgG}$ from patients with vitiligo compared with IgG that was not exposed to HCQ $(2,602.5 \pm 14.56$ vs. $2,962.00 \pm 35.68 ; \mathrm{P}<0.001$; Fig. 5).

$H C Q$ reduced the CDC activity of IgG obtained from vitiligo patients. The CDC activity was measured using the MTT assay. $\mathrm{IgG}$ (at concentration of 400 or $800 \mu \mathrm{g}$ ) obtained from patients with vitiligo significantly increased $\mathrm{CDC}$ activity compared with IgG obtained from controls $(\mathrm{P}<0.05$; Fig. 6). The addition of HCQ at $1 \mu \mathrm{g} / \mathrm{ml}$ significantly reduced the CDC activity when the serum IgG concentration was $>400 \mu \mathrm{g} / \mathrm{ml}(\mathrm{P}<0.05$; Fig. 6).

$H C Q$ reduced the $A D C C$ activity of $\operatorname{Ig} G$ obtained from vitiligo patients. The ADCC activity of purified IgG was measured using a lactate dehydrogenase-releasing assay. IgG obtained from patients with vitiligo significantly increased ADCC activity compared with IgG obtained from controls at different $\mathrm{E}$ :T ratios $(\mathrm{P}<0.05$; Fig. 6). The addition of HCQ at $1 \mu \mathrm{g} / \mathrm{ml}$ significantly reduced ADCC activity at an E:T ratio of 5:1 ( $\mathrm{P}<0.05$; Fig. 7). 
Table IV. Positive reactions between vitiligo patient and control serum samples and a collection of electrophoretic fractions obtained from an extract of human keratinocytes.

Electrophoretic fractions, $\mathrm{kDa}$

\begin{tabular}{|c|c|c|c|c|c|c|c|c|c|c|c|c|c|c|c|c|}
\hline Sample & $26-28$ & 30 & $33-35$ & $37-39$ & 42 & 50 & 53 & 60 & 65 & 68 & 75 & 85 & 90 & 100 & 110 & 126 \\
\hline \multicolumn{17}{|l|}{ Patients } \\
\hline 1 & & & & + & + & + & + & & & & & & + & & & \\
\hline 2 & & & + & + & & + & & & & & + & & + & + & & + \\
\hline 3 & + & & + & + & + & + & + & & + & & & & & & & \\
\hline 4 & + & & + & & & + & & & + & & & + & & + & & \\
\hline 5 & + & & + & & & + & & + & & & & & & + & & \\
\hline 6 & + & & + & & & + & + & & & + & + & & & & & \\
\hline 7 & + & & + & + & + & + & & & & & & & & & & \\
\hline 8 & + & & + & & & + & & & & & + & & & & & \\
\hline 9 & + & & + & + & + & + & & + & + & & & & & + & & \\
\hline 10 & + & & + & + & + & + & & & + & & & & + & & + & + \\
\hline 11 & + & & + & & & + & + & & & & + & + & + & & + & + \\
\hline 12 & + & & + & + & + & + & & & & & & & + & & & + \\
\hline 13 & + & & + & + & & + & & & & & & & + & & + & + \\
\hline 14 & & & & + & + & + & + & + & & & + & & & & + & \\
\hline 15 & & & + & + & & + & & & & & & & & & & \\
\hline \multicolumn{17}{|c|}{ Controls } \\
\hline 1 & + & & + & + & & + & & & & & & & & & & \\
\hline 2 & & & & & & + & & & & & + & & & & & \\
\hline 3 & & & & & & & & & & & & & & & & \\
\hline 4 & & & & & & & & & & & & & & & & \\
\hline 5 & & & & & & & & & & & & & & & & \\
\hline 6 & + & & + & + & & + & & & & & + & & & + & & + \\
\hline 7 & & & & & & & & & & & & & & & & \\
\hline 8 & + & & + & & & + & & & & & & & & & & \\
\hline 9 & & & & & & & & & & & & & & & & \\
\hline 10 & & & & & & & & & & & & & & & & \\
\hline 11 & & & & & & & & & & & & & & & & \\
\hline 12 & & & & & & + & & & & & & & & + & & \\
\hline 13 & & & & & & & & & & & & & & & & + \\
\hline 14 & + & & + & & & + & & & & & & & & & & \\
\hline 15 & & & & & & + & & & & & & & & & & \\
\hline
\end{tabular}

+ , indicates a positive reaction with the corresponding fraction.

\section{Discussion}

Previous studies support that autoimmunity is important in vitiligo pathogenesis $(7,8)$. However, the exact mechanism underlying this observation remains to be elucidated. Although cellular immunity has more frequently been the subject of investigation in recent years, humoral immunity has also been demonstrated to participate in the destruction of melanocytes, particularly in the process of extension $(7,8)$. In the present study, it was observed that serum concentrations of autoantibodies against melanocytes were higher in the majority of GV patients compared with controls, particularly in patients whose GV involved $>10 \%$ of the body surface. Notably, fluorescence staining for $\mathrm{IgG}$ indicated that $\mathrm{IgG}$ obtained from patient serum samples primarily bound to HMCs and HKCs and was often localized to the cytoplasm and membrane of the cells, only occasionally appearing in the nuclear region. In addition to exerting an effect on CDC and ADCC, these antibodies are likely to also have a role in increasing antigen uptake and presentation by dendritic cells. The present study used serum samples obtained from vitiligo patients, the autoantibody antigens were determined to have molecular weights of 30, 37-39, 42, 53, 60-75, 90, 100, 110 and $126 \mathrm{kDa}$, whereas the corresponding protein bands were rare in the control group. In addition, the majority of antigens were observed to be present in HMCs and HKCs, although the $30 \mathrm{kDa}$ antigen was observed only in the extracts of HMCs. These results are in agreement with those obtained in the immunofluorescence 
A

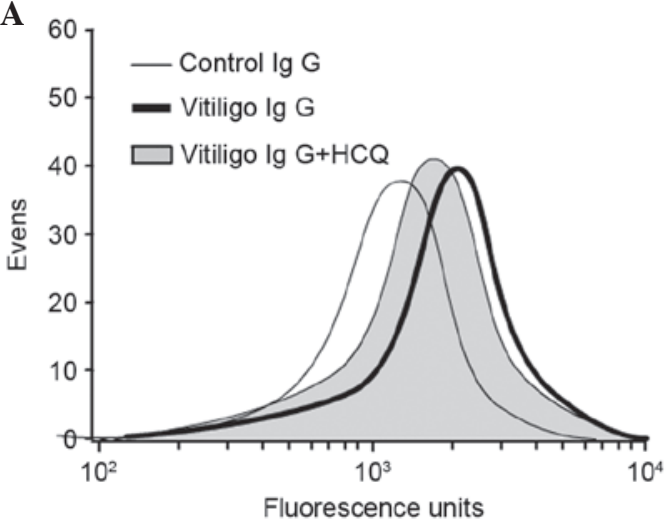

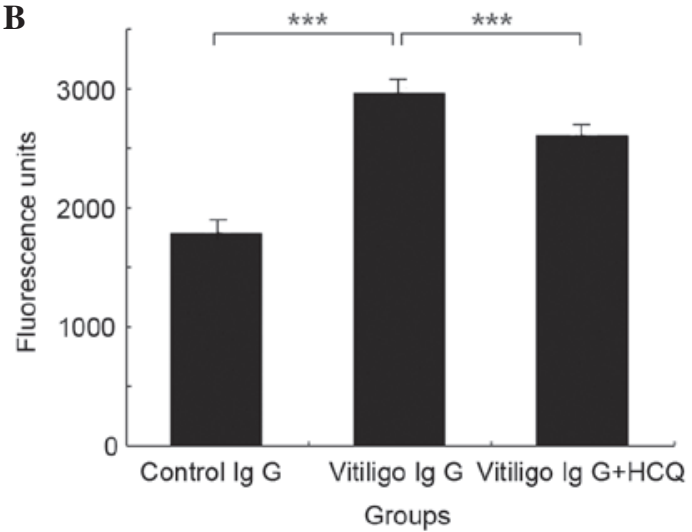

Figure 5. Effects of HCQ treatment on the binding of IgG (obtained from vitiligo patients) to melanocytes. Incubation of melanocytes with patient IgG samples resulted in significantly greater $\mathrm{IgG}$ binding compared with incubation with control $\mathrm{IgG}$ samples. The addition of $\mathrm{HCQ}$ at a concentration of $1 \mu \mathrm{g} / \mathrm{ml}$ significantly reduced the binding. (A) Flow cytometry detected fluorescence intensities between the different groups. (B) Mean fluorescence intensities of test groups. The data are presented as the mean \pm standard deviation $(\mathrm{n}=3)$. ${ }^{* * *} \mathrm{P}<0.001$. HCQ, hydroxychloroquine; IgG, immunoglobulin $\mathrm{G}$.

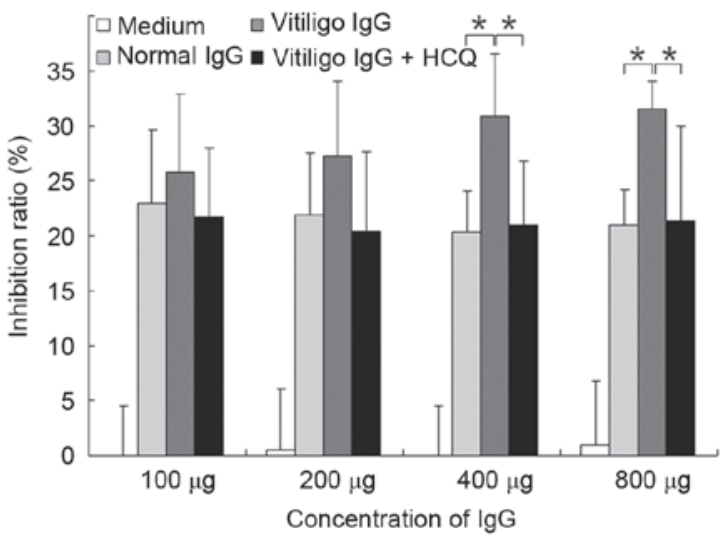

Figure 6. HCQ reduced the CDC activity of IgG samples taken from vitiligo patients. The incubation of melanocytes with patients IgG samples at different concentrations resulted in significantly greater CDC activity compared with incubation with control IgG samples. The addition of HCQ at a concentration of $1 \mu \mathrm{g} / \mathrm{ml}$ significantly reduced CDC activity. Data are presented as the mean \pm standard deviation $(\mathrm{n}=6)$. ${ }^{*} \mathrm{P}<0.05$. HCQ, hydroxychloroquine; $\mathrm{CDC}$, complement-dependent cytotoxicity; IgG, immunoglobulin G.

study. Candidate antigens for these reactions include tyrosinase, proto-oncogene C-kit, lysosomal-associated membrane protein-2, vitiligo (Vit)-40, Vit-75, and Vit-90 (9). These results support the hypothesis that melanocyte-specific antibodies and autoantibodies are important in the development of GV. However, it remains unknown why there appears to be selective destruction of HMCs in vitiligo instead of the destruction of HMCs and HKCs, as the same autoantigens are present in the two cell types.

Chloroquine (CQ) and HCQ are antimalarial therapeutic agents that are widely used in the immunosuppressive treatment of SLE and RA. The two molecules have similar structures and chemical properties, however, HCQ is safer and results in fewer side effects during treatment. In addition to their antimalarial action, the immunosuppressive effects of CQ and HCQ have been extensively investigated. Fox et al (10) reported that HCQ reduces proteolysis and antigen presentation by elevating lysosomal $\mathrm{pH}$. van den Borne et al (11) demonstrated that CQ and HCQ equally inhibit phytohemagglutinin-induced tumor

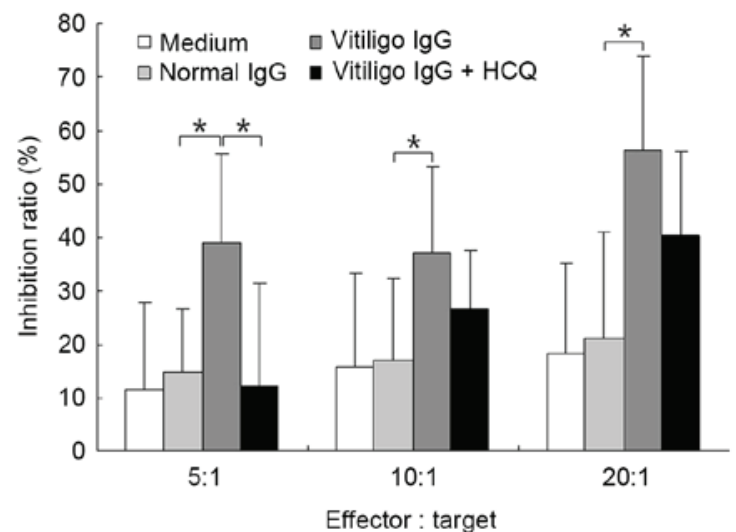

Figure 7. HCQ reduced the ADCC activity in IgG samples obtained from vitiligo patients. The incubation of melanocytes with patient $\mathrm{IgG}$ samples at a concentration of $400 \mu \mathrm{g} / \mathrm{ml}$ resulted in significantly greater ADCC activity compared with incubation with control IgG samples. The addition of HCQ at a concentration of $1 \mu \mathrm{g} / \mathrm{ml}$ significantly reduced ADCC activity. Data are presented as the mean \pm standard deviation $(n=6)$. ${ }^{*} \mathrm{P}<0.05$. HCQ, hydroxychloroquine; ADCC, antibody-dependent cell-mediated cytotoxicity; IgG, immunoglobulin G.

necrosis factor- $\alpha$ (TNF- $\alpha)$ and interferon- $\gamma$ production and lipopolysaccharide-induced TNF- $\alpha$ and interleukin- 6 production. Notably, previous studies have also demonstrated that CQ $(200 \mathrm{mg} / \mathrm{ml})$ could dissociate antigen-antibody complexes from red blood cells without leading to protein degeneration $(12,13)$. Rand et al (12) reported that HCQ (1 $\mu \mathrm{g} / \mathrm{ml})$ directly reduced the binding of antiphospholipid antibody- $\beta 2$-glycoprotein I complexes in vitro. These results implied that HCQ may alter antibody-antigen interactions and exert a beneficial effect on antibody-antigen reaction-induced autoimmune disease. The MTT assay was used to measure the cytotoxicity of HCQ. The results of the present study demonstrated that $1 \mu \mathrm{g} / \mathrm{ml}$ HCQ produced no cytotoxicity in HMCs. It is notable that $1 \mu \mathrm{g} / \mathrm{ml}$ is the mean blood concentration of HCQ in SLE patients undergoing HCQ treatment (14). The current study clearly demonstrates that HCQ reduced the binding of autoantibodies to HMCs in vitiligo patients and effectively decreased CDC and ADCC activity in response to the application of patient IgG. These results support the conclusion that HCQ 
may represent a promising therapeutic strategy for patients presenting with GV. However, further studies are required to examine whether HCQ produces clinical efficacy in GV patients.

In conclusion, the present study has provided preliminary evidence that HCQ dissociates antibody-antigen complexes and reverses the activities of ADCC and CDC in vitro. Additional studies are required to further elucidate the molecular mechanisms underlying these observations and the possible role of HCQ in the treatment of patients with progressive GV.

\section{Acknowledgements}

The present study was supported by the National Natural Science Foundation of China (grant nos. 81271745 and 81271774).

\section{References}

1. Guerra L, Dellambra E, Brescia S and Raskovic D: Vitiligo: Pathogenetic hypotheses and targets for current therapies. Curr Drug Metab 11: 451-467, 2010.

2. Njoo MD and Westerhof W: Vitiligo: Pathogenesis and treatment. Am J Clin Dermatol 2: 167-181, 2001.

3. Michelsen D: The double strike hypothesis of the vitiligo pathomechanism: New approaches to vitiligo and melanoma. Med Hypotheses 74: 67-70, 2010.

4. Furst DE: Pharmacokinetics of hydroxychloroquine and chloroquine during treatment of rheumatic diseases. Lupus 5 (Suppl 1): S11-S15, 1996.
5. Taïeb A and Picardo M; VETF Members: The definition and assessment of vitiligo: A consensus report of the Vitiligo European Task Force. Pigment Cell Res 20: 27-35, 2007.

6. Ma HJ, Yue XZ, Wang DG, Li CR and Zhu WY: A modified method for purifying amelanotic melanocytes from human hair follicles. J Dermatol 33: 239-248, 2006.

7. Cui J, Bystryn JC: Melanoma and vitiligo are associated with antibody responses to similar antigens on pigment cells. Arch Dermatol 131: 314-318, 1995

8. Yu HS, Kao CH and Yu CL: Coexistence and relationship of antikeratinocyte and antimelanocyte antibodies in patients with non-segmental-type vitiligo. J Invest Dermatol 100: 823-828, 1993.

9. Ruiz-Argüelles A, Brito GJ, Reyes-Izquierdo P, Pérez-Romano B and Sánchez-Sosa S: Apoptosis of melanocytes in vitiligo results from antibody penetration. J Autoimmun 29: 281-286, 2007.

10. Fox RI: Mechanism of action of hydroxychloroquine as an antirheumatic drug. Semin Arthritis Rheum 23 (2 Suppl 1): S82-S91, 1993.

11. van den Borne BE, Dijkmans BA, de Rooij HH, le Cessie S and Verweij CL: Chloroquine and hydroxychloroquine equally affect tumor necrosis factor-alpha, interleukin 6 , and interferon-gamma production by peripheral blood mononuclear cells. J Rheumatol 24: 55-60, 1997.

12. Rand JH, Wu XX, Quinn AS, Chen PP, Hathcock JJ and Taatjes DJ: Hydroxychloroquine directly reduces the binding of antiphospholipid antibody-beta2-glycoprotein I complexes to phospholipid bilayers. Blood 112: 1687-1695, 2008.

13. Holtz G, Mantel W, Buck W: The inhibition of antigenantibody reactions by chloroquine and its mechanism of action. Z Immunitatsforsch Exp Klin Immunol 146: 145-157, 1973.

14. Costedoat-Chalumeau N, Amoura Z, Hulot JS, Aymard G, Leroux G, Marra D, Lechat P and Piette JC: Very low blood hydroxychloroquine concentration as an objective marker of poor adherence to treatment of systemic lupus erythematosus. Ann Rheum Dis 66: 821-824, 2007. 